\title{
First passage time Markov chain analysis of rare events for kinetic Monte Carlo: double kink nucleation during dislocation glide
}

\author{
C S Deo ${ }^{1,2}$ and D J Srolovitz ${ }^{1}$ \\ ${ }^{1}$ Princeton Materials Institute \& Department of Mechanical and Aerospace Engineering, \\ Princeton University, Princeton, NJ 08544, USA \\ ${ }^{2}$ Department of Materials Science and Engineering, University of Michigan, Ann Arbor, \\ MI 48109, USA
}

Received 11 March 2002, in final form 6 July 2002

Published 20 August 2002

Online at stacks.iop.org/MSMSE/10/581

\begin{abstract}
We describe a first passage time Markov chain analysis of rare events in kinetic Monte Carlo (kMC) simulations and demonstrate how this analysis may be used to enhance kMC simulations of dislocation glide. Dislocation glide is described by the kink mechanism, which involves double kink nucleation, kink migration and kink-kink annihilation. Double kinks that nucleate on straight dislocations are unstable at small kink separations and tend to recombine immediately following nucleation. A very small fraction $(<0.001)$ of nucleating double kinks survive to grow to a stable kink separation. The present approach replaces all of the events that lead up to the formation of a stable kink with a simple numerical calculation of the time required for stable kink formation. In this paper, we treat the double kink nucleation process as a temporally homogeneous birth-death Markov process and present a first passage time analysis of the Markov process in order to calculate the nucleation rate of a double kink with a stable kink separation. We discuss two methods to calculate the first passage time; one computes the distribution and the average of the first passage time, while the other uses a recursive relation to calculate the average first passage time. The average first passage times calculated by both approaches are shown to be in excellent agreement with direct Monte Carlo simulations for four idealized cases of double kink nucleation. Finally, we apply this approach to double kink nucleation on a screw dislocation in molybdenum and obtain the rates for formation of stable double kinks as a function of applied stress and temperature. Equivalent kMC simulations are too inefficient to be performed using commonly available computational resources.
\end{abstract}

\section{Introduction}

The motion of a dislocation along its glide plane can be described by the kink mechanism (figure 1); kink nucleation, followed by kink migration and annihilation. Kinks nucleate and 
annihilate in pairs of opposite sense (i.e. double kinks). There is an energy barrier to dislocation motion, known as the Peierls barrier, which is associated with both the energy required to form a double kink and to move the individual kinks. Given the full energy landscape, it is, in principle, possible to predict the kinetics of dislocation migration within an absolute reaction rate theory framework.

The free energy $\Delta F$ of a double kink as a function of kink separation $w$ has the general form shown in figure 2 [1]. At some critical separation $w^{*}$, the attractive elastic interaction between two oppositely signed kinks is exactly balanced by an external force associated with an applied stress oriented to push the two kinks apart. The external force associated with the applied stress $\boldsymbol{\sigma}$ is simply $[\boldsymbol{b} \cdot \boldsymbol{\sigma} \times \boldsymbol{\xi}] \hat{\boldsymbol{b}} h$, where $\boldsymbol{b}$ is the Burgers vector of the dislocation, $h$ is the kink height, $\boldsymbol{\xi}$ is the dislocation line direction and the ${ }^{\wedge}$ indicates a unit vector. At $w^{*}$, the free energy of the pair is at its maximum $\Delta F^{*}$. A kink pair separated by more than $w^{*}$ will lower its free energy by further increasing the separation. Such a kink pair is unbounded. Opposite signed kinks separated by less than $w^{*}$ are attracted towards each other and will mutually annihilate. This description of annihilation and growth in terms of the free energy versus double kink size is equivalent to classical steady-state nucleation theory.

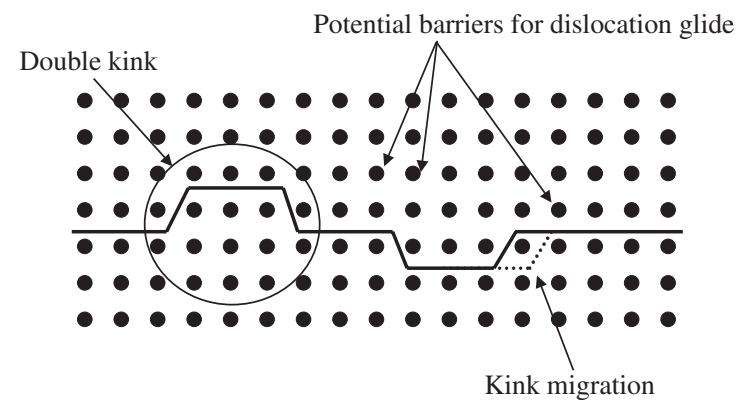

Figure 1. A schematic illustration of dislocation motion by the kink mechanism. The solid line is the dislocation. The circular dots indicate local maxima in the energy landscape (i.e. the Peierls barrier for dislocation glide). The dislocation moves across the Peierls barrier by nucleating kinks in pairs (double kinks). As the kinks migrate along the dislocation line, they meet and annihilate and the dislocation moves to the next Peierls valley.

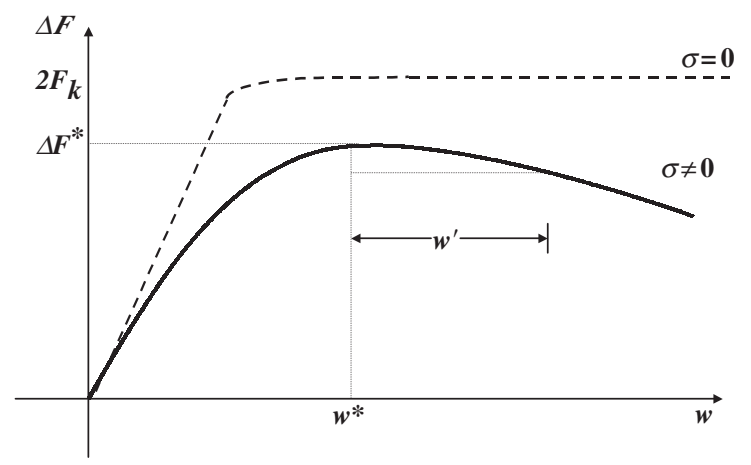

Figure 2. A schematic plot of the variation of the free energy $(\Delta F)$ of a double kink as a function of kink separation distance $(w)$. The dotted and solid curves represent the free energy profile in the absence and presence of an applied stress. The free energy of the double kink exhibits a maximum $\Delta F^{*}$ at a kink separation $w^{*} . w^{\prime}$ is the distance beyond $w^{*}$, where the free energy of the double kink is $\Delta F^{*}-k T$. 
Nucleation theory can be applied to the double kink problem to obtain the effective width of the saddle point and hence the width of a stable double kink. Let us define $w^{\prime}$ as the distance beyond $w^{*}$ where the free energy of the double kink is $\Delta F^{*}-k T$, where $k T$ is the thermal energy. Kink pairs of a width greater than $w^{*}+w^{\prime}$ have a negligible probability of self-annihilation, since this would require an energy larger than $k T$ to move the kinks back over the saddle point. Kink pairs of width greater than $w^{*}$ but less than $w^{*}+w^{\prime}$ have not passed the activation barrier yet, because a fraction of them can diffuse against the applied stress, annihilate and never contribute to the net motion of the dislocation.

Kinetic Monte Carlo (kMC) methods provide an ideal approach for simulating dislocation migration in terms of the kink model $[2,3]$. In a kMC simulation of dislocation migration, the rates at which the kinks nucleate and move are calculated from the energy landscape and the kinks are moved in accordance with these rates. Before the dislocation can move, however, we must first nucleate the double kinks. Unfortunately, nucleating a stable double kink can be very slow in a kMC simulation since when $w$ is smaller than $w^{*}$ only exponentially few sub-critical double kinks ever grow to reach $w>w^{*}$. In fact, the vast majority of double kinks nucleated will self-annihilate before they reach the critical size. An alternative approach to avoid this vast number of wasted Monte Carlo steps is to nucleate the double kinks with a sufficiently large width $\left(>\left(w^{*}+w^{\prime}\right)\right)$ to avoid most of the double kink self-annihilation events. In doing so, we must properly account for the time it would have taken the standard kMC simulation to actually nucleate such a stable double kink.

Several researchers have addressed the issue of the long times required for double kink nucleation in their kMC models of dislocation glide [4-7]. In the pioneering kMC simulations of dislocation glide performed by Lin and Chrzan [5] and Tang et al [6], several ad hoc assumptions were made about the nucleation rates of double kinks of fixed separation. Lin and Chrzan assumed that the nucleation rate is proportional to $\exp \left(-E_{\mathrm{b}} / k T\right)$, where $E_{\mathrm{b}}$ is the barrier height for double kinks of width $20 \mathrm{~b}$. Tang et al assumed that the double kink nucleation rate is inversely proportional to the kink separation (which they fixed at $15 b$ ). This approach does not account for the fact that a double kink can form with separations smaller than the critical size and then grow to the critical size or self-annihilate. This process should clearly depend on the magnitude of the applied stress and the temperature (neither of which were explicitly included in these analyses). Cai et al [4] addressed this issue by treating the formation of a critical double kink as a Markov process on the discrete width of the double kink separation. They calculated the probability that a double kink reaches the critical width without self-annihilating and used this survival probability to compute the nucleation rate of a stable double kink. While this is an advance over the earlier approaches, it does not account for the time that the double kink spends at sub-critical kink separations.

In this paper, we extend the Markov process analysis of Cai et al [4] to determine the true rate of nucleation of a stable double kink by including the full energy landscape the double kink sees as it grows and the time it spends at each sub-critical width. This approach yields the distribution of times required for a double kink to achieve the stable kink separation. The theoretical predictions of the nucleation rate are tested against full Monte Carlo simulations. Finally, we examine several approximations that can be used to efficiently model arbitrary energy landscapes such that this double kink nucleation model can be incorporated directly into kMC simulations of dislocation motion.

\section{Double kink nucleation as a Markov process}

We consider double kink nucleation as a temporally homogeneous birth-death Markov process; i.e. a discrete state jump Markov process in which the local jump probabilities are time invariant 
and the double kink spacing can increase by 1 (birth) or decrease by 1 (death). Markov processes and random walks $[8,12,13]$ can be used to describe several physical phenomena and their characteristics are well studied. In this model, a double kink is nucleated with separation 1, in some units, and this separation can change by \pm 1 in any step. This is a one-dimensional model where the single variable is the discrete double kink separation.

Any discrete state jump Markov process $X(t)$ (the double kink separation here) can be described by the following characterizing functions in the manner of Gillespie [8]:

(a) $a(n, t) \mathrm{d} t$ is the probability that the process will jump away from state $n$ in the next infinitesimal time interval $[t, t+\mathrm{d} t]$,

(b) $w(v \mid n, t) \mathrm{d} t$ is the probability that the process, upon jumping away from state $n$ at time $t$ will land in state $n+v$ at time $t+\mathrm{d} t$,

where $n=X(t)$ and $n$ and $v$ are integers. The Markov process is temporally homogeneous if the characterizing functions do not change with time. Since $X(t)$ is temporally homogeneous, $a(n, t)=a(n)$ and $w(v \mid n, t)=w(v \mid n)$.

A temporally homogeneous birth-death process $X(t)$ can be defined as any discrete state jump Markov process whose characterizing functions $a(n, t)$ and $w(v \mid n, t)$ have the respective forms

$$
\begin{aligned}
& a(n, t)=a(n), \quad(n \geqslant 0), \\
& w(v \mid n, t)= \begin{cases}w_{+}(n), & \text { if } v=+1 \text { and } n \geqslant 0, \\
w_{-}(n), & \text { if } v=-1 \text { and } n \geqslant 0, \\
0, & \text { otherwise. }\end{cases}
\end{aligned}
$$

Thus the Markov process $X(t)$ at any state $i$, has only two states accessible to it; $i-1$ and $i+1$. The consolidated characterizing function $W(v \mid n, t)$ can be defined as the product of $a(n, t)$ and $w(v \mid n, t)$

$$
W(v \mid n, t)=W_{ \pm}(v \mid n)= \begin{cases}W_{+}(n)=a(n) w_{+}(n), & \text { if } v=+1 \text { and } n \geqslant 0, \\ W_{-}(n)=a(n) w_{-}(n), & \text { if } v=-1 \text { and } n \geqslant 0, \\ 0, & \text { otherwise, }\end{cases}
$$

where $W_{+}(n)$ and $W_{-}(n)$ are the stepping functions of the process or the probability that the system at state $n$ will jump to $n+1$ and $n-1$ in time $\mathrm{d} t$. The nature of the characterizing functions for the Markov process that describes double kink nucleation is described in the following section.

\section{Simulation of the double kink nucleation as a one-dimensional random walk}

The double kink nucleation can be simulated as a one-dimensional random walk on the discrete, non-negative integer kink separations. The random walk is biased by the nature of the dependence of the free energy on the kink separation as shown in figure 3 . There is an activation barrier associated with the migration of the individual kinks (see figures 1 and 3), known as the secondary Peierls barrier to distinguish it from the Peierls barrier associated with translating the entire dislocation perpendicular to itself in its glide plane. As described in the previous section, discrete non-negative values of the kink separation correspond to distinct Markov states for the one-dimensional random walk. Thus, the double kink separation $i$ can change to state $i+1$ with a rate $\lambda_{i}$ and to state $i-1$ with a rate $\mu_{i}$ given by the expressions below:

$$
\begin{aligned}
\lambda_{i} & =v \exp \left(-\frac{Q_{i}^{+}}{k_{\mathrm{B}} T}\right), \\
\mu_{i} & =v \exp \left(-\frac{Q_{i}^{-}}{k_{\mathrm{B}} T}\right) .
\end{aligned}
$$



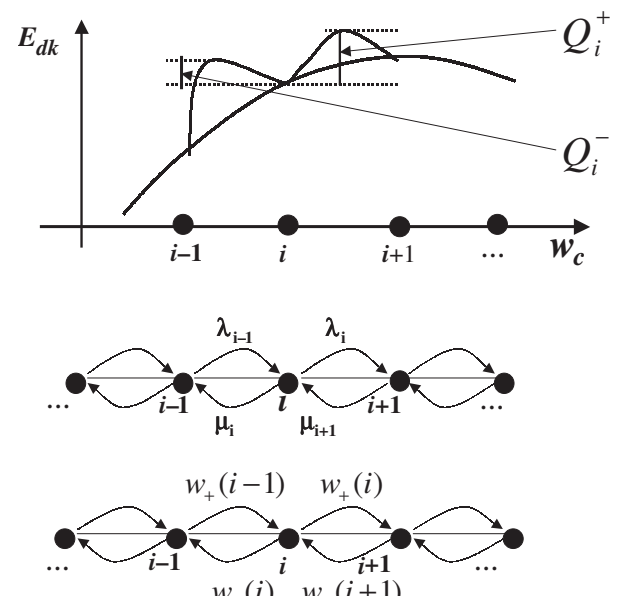

$w_{-}(i) \quad w_{-}(i+1)$

Figure 3. A schematic illustration of the nucleation of a double kink treated as a birth-death Markov process. The figure shows an arbitrary double kink energy landscape, which is a plot of the double kink energy as a function of the kink separation distance. The rates at which the double kink increases or decreases in size by a unit Burgers vector can be obtained from the energy landscape (see equations (3)) and used to compute the forward and backward jump probabilities of the Markov process at each state (see equations (4)).

The pre-factor $v$ is the rate at which the kink attempts to migrate (associated with the vibrational spectrum of the solid). The probability that the double kink separation will go from $i$ to $i+1$ is $w_{+}(i)$, and to state $i-1$ is $w_{-}(i)$, as shown in figure 3 , where the characterizing functions $w_{+}(i)$ and $w_{-}(i)$ were defined for the Markov process $X(t)$ in the previous section:

$$
\begin{aligned}
& w_{+}(i)=\frac{\lambda_{i}}{\lambda_{i}+\mu_{i}}, \\
& w_{-}(i)=\frac{\mu_{i}}{\lambda_{i}+\mu_{i}} .
\end{aligned}
$$

The simulation is started with the random walker in state $i=0$. This corresponds to the Markov process $X(t)=0$, where no double kink is present. From any state $i(i>0)$, the random walker is allowed to jump to states $i+1$ or $i-1$ with probabilities $w_{+}(i)$ and $w_{-}(i)$, respectively. From $i=0$, the random walker moves to $i=1$ with probability 1 . The direction of the jump is determined by sampling a random number $\zeta$ from $[0,1)$. If $\zeta<w_{+}(i)$, the random walker moves to state $i+1$; if not, it moves to state $i-1$.

The time that the random walker spends in a state before it makes the next move is exponentially distributed over the sum of the rates of all possible jumps from that state. On average, the time spent in state $i$ is $1 /\left(\lambda_{i}+\mu_{i}\right)$. This is the inverse of the characterizing function $a(i)$ of the Markov process $X(t)$, defined in the previous section:

$$
a(i)=\lambda_{i}+\mu_{i}
$$

This simple simulation procedure captures the nature of embryonic double kink nucleation and growth. Using this procedure, it is possible to determine the time it would take a double kink to reach a stable kink separation by repeating this simulation many times to obtain an average time for the kink separation to reach a pre-determined size. However, it is not practical to do so in the case of many real materials. For small kink separations in real systems, the forward probabilities are very small (such as in molybdenum for which $w_{+}(i)<0.001$ for $i=0,1,2[7,9])$. Thus, in this simple simulation, the random walker gets trapped at small kink 
separations and would never reach the critical separation during easily realizable simulations. Therefore, this method is not a practical approach to determining double kink nucleation rates. There are two approaches to overcome this difficulty: (1) by refining the MC simulation procedure using importance sampling techniques and (2) by analysing the Markov process to obtain first passage $[8,12,13]$ (or first crossing) times for the double kink to reach a critical kink separation. We follow the second approach below.

\section{First passage time analysis}

If a temporally homogeneous birth-death process $X(t)$ is placed in some specified state $n_{0}$ at time $t=0$, the first passage (or first crossing) time $T\left(n_{0} \rightarrow n_{1}\right)$ is the time of the first arrival of the process in state $n_{1}$. In the case of the double kink nucleation process, we are interested in the case where $n_{0}$ corresponds to the absence of the double kink $\left(n_{0}=0\right)$ and $n_{1}$ corresponds to the presence of a stable double kink (i.e. one with a kink separation greater than $w^{*}+w^{\prime}$ ) as shown in figure 1 . The exact value of $n_{1}$ depends on the energy landscape for double kink nucleation and growth.

The first passage time $T\left(n_{0} \rightarrow n_{1}\right)$ is a random variable. We denote its density function by $Q\left(t ; n_{0} \rightarrow n_{1}\right)$ and its $k$ th moment by $T_{k}\left(n_{0} \rightarrow n_{1}\right)$. The moments can be calculated from the density function as

$$
T_{k}\left(n_{0} \rightarrow n_{1}\right)=\int_{0}^{\infty} t^{k} Q\left(t ; n_{0} \rightarrow n_{1}\right) \mathrm{d} t .
$$

For any temporally homogeneous birth-death process, Gillespie [8] has shown that the distribution of the first passage time $Q\left(t, n_{0} \rightarrow n_{1}\right)$ can be obtained by computation of the eigenvalues of an $(m \times m)$ matrix $\boldsymbol{M}$ where $m=n_{1}+1$ is the number of states accessible to the process in the course of a first passage from state $n_{0}=0$ to state $n_{1}$. The matrix $\boldsymbol{M}$ is tri-diagonal with elements that are the characterizing function $a(n)$ and the stepping function $W_{ \pm}(i)$ of the Markov process $X(t)$ :

$M=\left(\begin{array}{cccccccc}a(0) & -W_{-}(1) & 0 & \ldots & \ldots & \ldots & \ldots & 0 \\ -W_{+}(0) & a(1) & -W_{-}(2) & \ldots & \ldots & \ldots & \ldots & \ldots \\ 0 & -W_{+}(1) & a(2) & \ldots & \ldots & \ldots & \ldots & \ldots \\ \ldots & 0 & -W_{+}(2) & \ldots & \ldots & \ldots & \ldots & \ldots \\ \ldots & \ldots & 0 & \ldots & \ldots & \ldots & 0 & \ldots \\ \ldots & \ldots & \ldots & \ldots & \ldots & \ldots & -W_{-}\left(n_{1}-1\right) & 0 \\ \ldots & \ldots & \ldots & \ldots & \ldots & \ldots & a\left(n_{1}-1\right) & 0 \\ \ldots & \ldots & \ldots & \ldots & \ldots & \ldots & -W_{+}\left(n_{1}-1\right) & 0\end{array}\right)$,

where we recall that $a(n)=W_{-}(n)+W_{+}(n)$. In the case of double kink nucleation, the functions $a(i)$ and $W_{ \pm}(i)$ are the characterizing function and the stepping functions, respectively, that are obtained from the energy landscape by using equations (2)-(5).

The density function for the first passage time for the case $n_{0}<n_{1}$ is given by the relation [8]

$$
Q\left(t: n_{0} \rightarrow n_{1}\right)=\sum_{i=1}^{n} C_{i}\left(n_{0}, n_{1}\right) \lambda_{i} \exp \left(-\lambda_{i} t\right) .
$$

Here $\lambda_{1}, \lambda_{2}, \lambda_{3}, \ldots, \lambda_{n_{1}}$ are the $n_{1}$ non-zero eigenvalues of the matrix $\boldsymbol{M}$. The coefficients $C_{i}\left(n_{0}, n_{1}\right)$ are obtained from the elements of the canonical matrix $\boldsymbol{V}$ of matrix $\boldsymbol{M}$ (the columns of the matrix $\boldsymbol{V}$ are the eigenvectors of the matrix $\boldsymbol{M}$ ):

$C_{i}\left(n_{0}, n_{1}\right)=-(V)_{n_{1}+1, i+1}\left(V^{-1}\right)_{i+1, n_{0}+1}, \quad\left(n_{0}<n_{1} ; i=1,2,3, \ldots, n_{1}\right)$. 
The $k$ th moment of $Q\left(t ; n_{0} \rightarrow n_{1}\right)$ can be obtained by using equation (6) and the expression for $Q\left(t ; n_{0} \rightarrow n_{1}\right)$ (equation (8)):

$$
T_{k}\left(n_{0} \rightarrow n_{1}\right)=k ! \sum_{i=1}^{n_{1}} \frac{C_{i}\left(n_{0}, n_{1}\right)}{\lambda_{i}^{k}}, \quad\left(n_{0}<n_{1} ; k \geqslant 0\right)
$$

The average first passage time $T_{1}\left(n_{0} \rightarrow n_{1}\right)$ is the first moment of $Q\left(t ; n_{0} \rightarrow n_{1}\right)$ :

$$
T_{1}\left(n_{0} \rightarrow n_{1}\right)=\sum_{i=1}^{n_{1}} \frac{C_{i}\left(n_{0}, n_{1}\right)}{\lambda_{i}}, \quad\left(n_{0}<n_{1}\right) .
$$

In the case of the double kink nucleation the density function and the average of the first passage time for the double kink to reach a stable kink separation is given by

$$
\begin{aligned}
& Q\left(t: 0 \rightarrow n_{1}\right)=\sum_{i=1}^{n_{1}} C_{i}\left(0, n_{1}\right) \lambda_{i} \exp \left(-\lambda_{i} t\right), \\
& T_{1}\left(0 \rightarrow n_{1}\right)=\sum_{i=1}^{n_{1}} \frac{C_{i}\left(0, n_{1}\right)}{\lambda_{i}},
\end{aligned}
$$

with

$$
C_{i}\left(0, n_{1}\right)=-(V)_{n_{1}+1, i+1}\left(V^{-1}\right)_{i+1,1}, \quad\left(i=1,2,3, \ldots, n_{1}\right) .
$$

An alternative expression can also be derived for the average first passage time $T_{1}\left(n_{0} \rightarrow n_{1}\right)$ based on a recursion relation $[8,10]$. The derivation of this relation is more intuitive and it can be used for more computationally efficient calculation of the average first passage time. The average first passage time is simply the sum of the average time that the Markov process spends in each state:

$$
T_{1}\left(n_{0} \rightarrow n_{1}\right)=\sum_{n=0}^{n-1} t\left(n ; n_{0} \rightarrow n_{1}\right), \quad\left(n_{0}<n_{1}\right) .
$$

The average time, $t\left(n ; n_{0} \rightarrow n_{1}\right)$, spent in each state $n$ during the process of going from $n_{0}$ to $n_{1}$ is obtained from the following recursive relationship:

$$
\begin{aligned}
& t\left(n_{1}-1 ; n_{0} \rightarrow n_{1}\right)=\frac{1}{W_{+}\left(n_{1}-1\right)}, \quad\left(n_{0}<n_{1}\right), \\
& t\left(n ; n_{0} \rightarrow n_{1}\right)=\frac{\Theta\left(n+1-n_{0}\right)}{W_{+}(n)}+\frac{W_{-}(n+1)}{W_{+}(n)} t\left(n+1 ; n_{0} \rightarrow n_{1}\right),
\end{aligned}
$$

where $\Theta(j)$ is the Heaviside step function. Thus, equation (13) provides a method to calculate the average first passage time $T_{1}\left(n_{0} \rightarrow n_{1}\right)$ using the recursive relationships from equations (14a) and (14b). As before, for double kink nucleation $n_{0}=0$, and $n_{1}$, the characterizing function $a(n)$ (equations (3) and (5)) and the stepping functions $W \pm(n)$ (equation (2)) depend on the energy landscape.

We have described two ways of calculating the average first passage time for a Markov process to reach a certain state. The first approach is comprehensive and leads to the computation of the entire density function along with all the moments of the first passage times. The second approach is much more computationally efficient but only yields the average first passage time.

In the case of a Markov process that describes kink nucleation, we are interested in the time that the process takes to reach a state that corresponds to stable kink separation. For some simple energy landscapes, the simulation procedure described in the previous section can also be used to estimate this time. In the next section, we compare the results of the simulation procedure and the first passage analysis for some simple cases of double kink nucleation. Then, we discuss the application of this process to dislocation motion. 


\section{Idealized energy landscape}

We now apply the first passage time analyses, discussed in the previous section, to four idealized cases of double kink nucleation, before turning to the more important case of double kink nucleation on a screw dislocation in molybdenum. In these idealized cases, we compare the first passage time predictions with the results of direct Monte Carlo random walker simulations. Comparisons are made for both the full eigenvalue approach (equations (12)) and the recursion approach (equations (13) and (14)).

For each case, there is an activation barrier (secondary Peierls barrier-the horizontally spaced circles in figure 1) associated with the migration of kinks. The simulation is started with no kinks present, corresponding to $X(0)=0$. At each step, a random number $\zeta$ is sampled from [0,1). If $\zeta<w_{+}(i)$, the Markov process jumps to state $i+1$, if not it jumps to $i-1$ (the Peierls barrier height only scales time). For simplicity, we assume that the time the Markov process spends in each state that it accesses is unity, in some arbitrary time units, i.e. $a(i)=\left(\lambda_{i}+\mu_{i}\right)=1$. As the simulation proceeds, we record the first passage time for each accessible state. The simulation is terminated when the double kink reaches a kink separation distance of $15 b$, i.e. the Markov process has the following accessible states: $0,1,2, \ldots, 15$. We repeat the simulation $10^{4}$ times to determine the distribution of first crossing times and the average first crossing times for each state.

\subsection{Case 1: double kink energy does not vary with kink separation}

We first consider the simplest possible case; that is, the energy of the double kink is independent of the state in which the double kink resides, as shown in the free energy-kink separation plot in figure 4(a). In this case, the Markov process at any state $i$ has equal probability of jumping to states $i+1$ or $i-1$ (i.e. $\left.w_{+}(i)=w_{-}(i)=0.5\right)$.

The distribution of the first passage times for the Markov process to reach the final state $Q(t ; 0 \rightarrow 15)$ is shown in figure $4(b)$. The maximum in the distribution of first passage times occurs at approximately $70 \tau$, where $\tau$ is the reduced unit of time. The tail of the distribution is very long because many of the double kinks that form and grow, selfannihilate before they reach the critical size. Because of this long tail (even on a logarithmic scale), the average first crossing time is much longer than the most probable first crossing time. In the present case, the average of the first passage time $(225 \tau)$ coincides with the square of the number of Markov states between $i=0$ and $i=w_{\mathrm{c}}(=15)$. This is not surprising as the Markov process is a random walker on a level energy surface and, hence, in the long time limit its evolution is well-described by a one-dimensional diffusion equation (i.e. the mean squared displacement of the random walker is proportional to the number of random walk steps taken).

Figure $4(c)$ shows the average first passage time $T_{1}(0 \rightarrow n)$ taken by the Markov process $X(t)$ to reach each accessible state $(n=1, \ldots, 15)$ from the initial state $n_{0}=0$ for each state $n$. We compare the results of the analytical approaches (the eigenvalue approach and the recursive formula) and the simulation procedure. In this case (equal forward and back probabilities), the results of the two analyses coincide exactly and are in excellent agreement with the Monte Carlo simulation results. As in the results in figure $4(b)$, for $n=15$, the average first passage time to reach each state is given by the square of its distance from the initial state.

This parabolic dependence can be obtained analytically from equation (13) and the recursion relations in equations (14) for the special case in which the probabilities are independent of $i$ for all $i>0$, i.e. $w_{+}(i)=f$ and $w_{-}(i)=b$. This is the situation in 
(a)
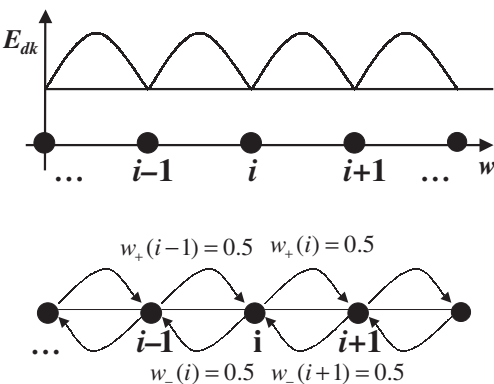

(b)

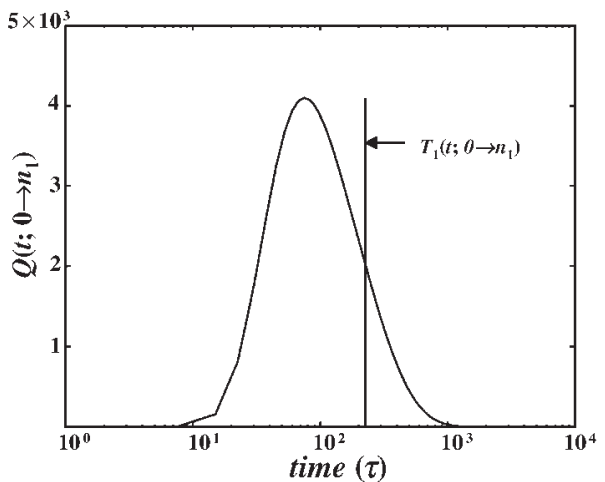

(c)

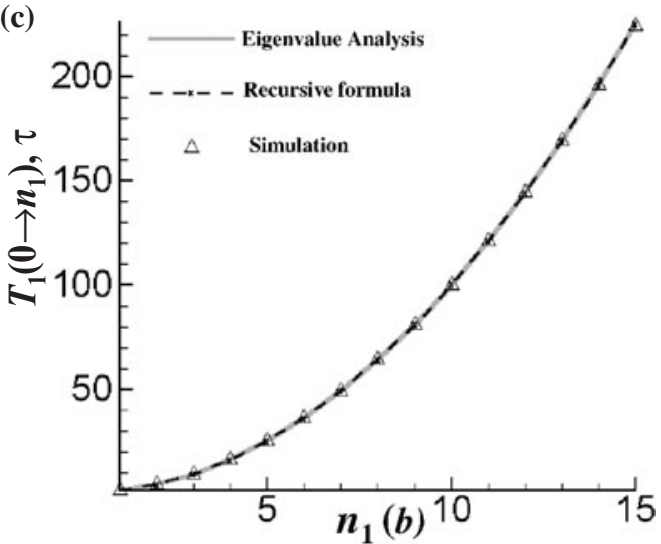

Figure 4. Idealized case 1: (a) The double kink energy separation relation for the case in which the energy of the double kink is independent of kink separation. In this case, the probability of the double kink growing or shrinking by a unit width is 0.5 for all kink-kink separations. (b) The distribution of first passage times for the double kink to reach a width of $15 b$; the vertical line indicates the average first passage time $T_{1}\left(0 \rightarrow n_{1}\right)$. (c) The average first passage times $T_{1}(0 \rightarrow n)$ for the double kink to reach a width $n_{1}$. The solid grey line, dotted black line and triangles represent the results of the eigenvalue approach, the recursion formula, and the Monte Carlo simulations, respectively.

the present case, case 1 , and cases 2 and 3 (figures $4(a), 5(a), 6(a)$ ). The average first passage time can be written as

$$
\begin{aligned}
T_{1}\left(0 \rightarrow n_{1}\right) & =\sum_{i=0}^{n_{1}-1} \alpha^{i}+\frac{1}{f} \sum_{j=1}^{n_{1}-1} \sum_{i=0}^{n_{1}-1-j} \alpha^{i} \\
& =\frac{n_{1}}{f}\left(\sum_{i=0}^{n_{1}-1} \alpha^{i}\right)-\frac{1}{f}\left(\sum_{i=0}^{n_{1}-1}(i+1) \alpha^{i}\right)+\left(\sum_{i=0}^{n_{1}-1} \alpha^{i}\right),
\end{aligned}
$$

where $\alpha=W_{-}(i+1) / W_{+}(i)=w_{-}(i+1) w_{+}(i)=b / f$. For the case described by figure $4(a)$, $\alpha=1$ and $f=0.5$, which, when inserted in equation (15), leads to $T_{1}\left(0 \rightarrow n_{1}\right)=n_{1}^{2}$. This is in agreement with the results presented in figure $4(c)$.

\subsection{Case 2: double kink energy increases with increasing kink separation}

Now, consider double kink nucleation such that the double kink energy increases linearly with increasing kink separation distance. The energy-kink separation relationship is plotted in 
(a)

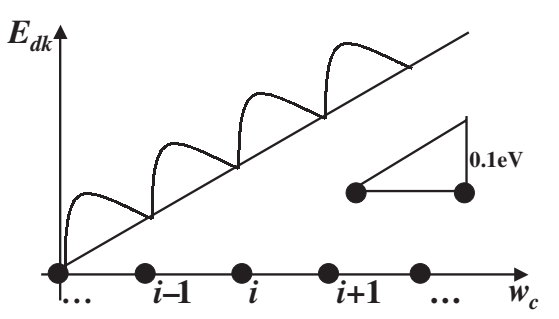

(b)

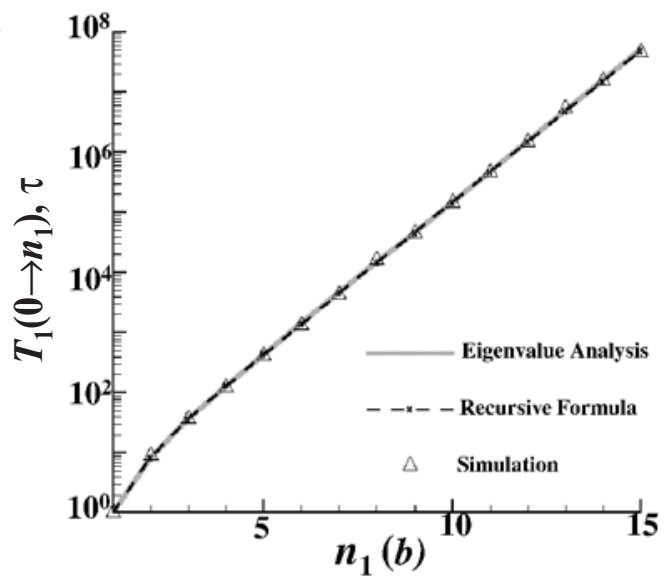

Figure 5. Idealized case 2: (a) the double kink energy separation relation for the case in which the energy of the double kink increases linearly with the double kink width. At any state $i$ the probability to move forward (to state $i+1$ ) is less than the probability to move backward (to state $i-1)$. (b) The average first passage times $T_{1}\left(0 \rightarrow n_{1}\right)$ for the double kink to reach width $n_{1}$, where the lines and symbols have the same meaning as in figure $4(c)$.

figure 5(a). From each state $i$, the Markov process $X(t)$ can jump to states $i+1$ or $i-1$ with probabilities $w_{+}(i)$ and $w_{-}(i)$ defined by equations $(4 a)$ and $(4 b)$. In this case, $w_{+}(i)<w_{-}(i)$ for each state.

The average first passage time for the Markov process to reach each accessible state $(n=1-15)$ from the initial state $n_{0}=0$ is $T_{1}(0 \rightarrow n)$ and can be obtained directly using the eigenvalue (equations (12)) and the recursive (equation (13)) approaches. In figure 5(b), we compare the average first passage time obtained from the two analytical approaches and from the simulation procedure for each accessible state $(n=1-15)$.

For large $n, T_{1}(0 \rightarrow n)$ increases exponentially with increasing $n$. As for the energy profile in figure 4, there is excellent correspondence between the two analytical results and the Monte Carlo simulation results. However, in the present case, the two analytical results are not in exact agreement (although the agreement appears nearly perfect in figure $5(b)$ ). Since the double kink energy increases with increasing kink separation, the two kinks attract at all kink separation distances. Thus, the Markov process $X(t)$ always has higher probability of travelling backwards (towards state 0 ). This explains why, in this case, it takes much longer for the Markov process $X(t)$ to reach a particular state than in the case where there is no interaction between kinks (case 1). Note that in this case, the double kink is never stable, since the lowest energy configuration is one with no double kinks (state $n_{0}=0$ ).

The results in figure $5(b)$ can be understood by reference to equation (15) for the special case in which the double kink energy increases linearly with the kink separation. This implies a constant value of $\alpha>1$. We rewrite the expression for the average passage time equation (15) as

$T_{1}\left(0 \rightarrow n_{1}\right)=\alpha^{n_{1}-1}+\alpha^{n_{1}-2}\left(1+\frac{1}{f}\right)+\alpha^{n_{1}-3}\left(1+\frac{2}{f}\right)+\cdots+\alpha^{0}\left(1+\frac{n_{1}-1}{f}\right)$.

The first term dominates for large values of $n_{1}$ and $\alpha>1$. This suggests that for large $n_{1}, T_{1}$ increases exponentially with $n_{1}$. This is consistent with the results plotted in figure $5(b)$ for $\alpha=3.2$ and $f=W_{+}(i)=w_{+}(i)=0.238$. In this case, the exponential approximation is accurate for $n_{1}>3$. 


\subsection{Case 3: double kink energy decreases with increasing kink separation}

The third case examines the situation where the double kink energy decreases with increasing kink separation. The energy-kink separation relationship is shown in figure $6(a)$. The probabilities, $w_{+}(i)$ and $w_{-}(i)$, defined in equations $(4 a)$ and $(4 b)$, are such that $w_{+}(i)$ is greater than $w_{-}(i)$ for every state $i$ accessible to the Markov process.

Figure $6(b)$ compares the average first passage time results from the two analytical approaches with the Monte Carlo simulation. We see that $T_{1}\left(0 \rightarrow n_{1}\right)$ increases with increasing $n_{1}$ in a nearly linear manner. There is excellent correspondence between the two analytical results and the Monte Carlo simulation results over the entire range of $n_{1}$. The free energy of the double kink decreases with increasing kink separation. Thus, at every state, the kinks repel each other. Therefore, the Markov process $X(t)$ always has higher probability of travelling forward (away from state 0 ). This results in the Markov process $X(t)$ taking a very short time to reach large $n_{1}$ states compared with results of cases 1 and 2 . Note that in this case the double kink is always stable, since the highest energy configuration is the one with no double kinks (state $\left.n_{0}=0\right)$.

We can also analyse the present situation in which the energy decreases with increasing kink separation using equation (15). It is convenient to rewrite equation (15) for the constant $\alpha<1$ case as

$$
\begin{aligned}
T_{1}\left(0 \rightarrow n_{1}\right) & =\left(1+\frac{n_{1}-1}{f}\right)+\alpha^{1}\left(1+\frac{n_{1}-2}{f}\right)+\alpha^{2}\left(1+\frac{n_{1}-3}{f}\right)+\cdots+\alpha^{n_{1}-1} \\
& \approx \frac{-2 \alpha}{(1-\alpha)^{2}}+\left(\frac{1+\alpha}{1-\alpha}\right) n_{1},
\end{aligned}
$$

where the second expression is valid for large $n_{1}$ and $\alpha<1$ and where we have substituted $f=(1+\alpha)^{-1}$. This result demonstrates that, for $n_{1}$ sufficiently large, $T_{1}$ is a linear function of $n_{1}$, which is consistent with the results in figure $6(b)$. A linear regression analysis of the data in figure $6(b)$ (for $\alpha=0.312$ ) suggests that $T_{1}=-1.17+1.90 n_{1}$ while the second expression in equation (18) gives $T_{1}=-1.32+1.91 n_{1}$. Therefore, the second expression in equation (18) is

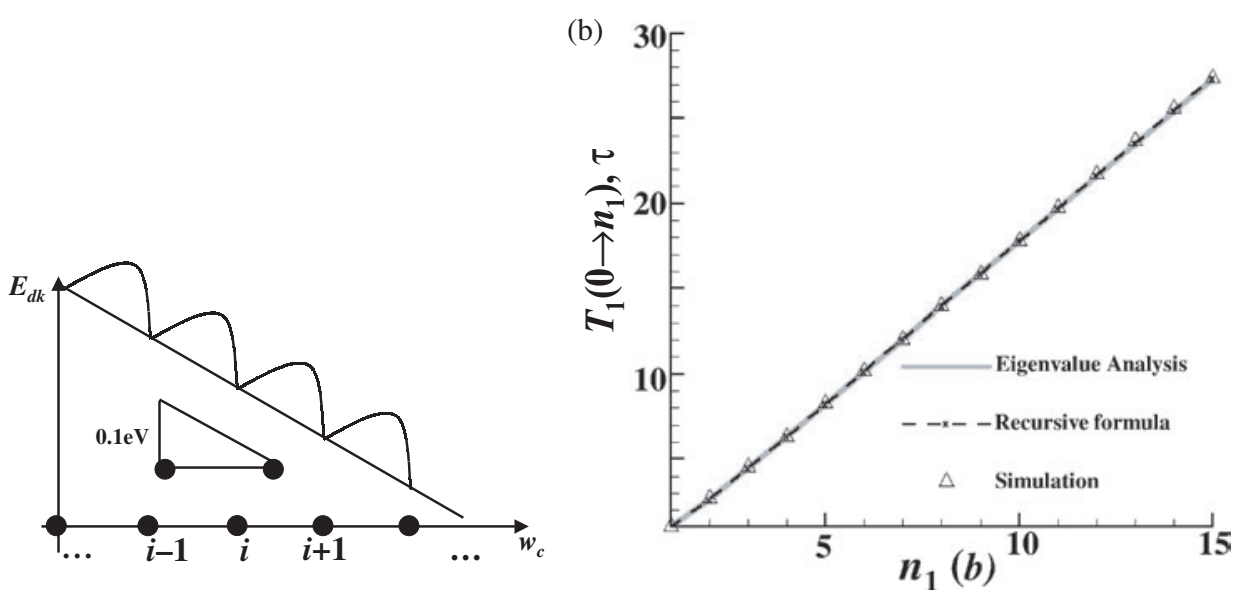

Figure 6. Idealized case 3: (a) the double kink energy separation relation for the case in which the energy of the double kink decreases linearly with the double kink width. At any state $i$ the probability to move forward (to state $i+1$ ) is greater than the probability to move backward (to state $i-1)$. (b) The average first passage times $T_{1}\left(0 \rightarrow n_{1}\right)$ for the double kink to reach width $n_{1}$, where the lines and symbols have the same meaning as in figure $4(c)$. 
(a)

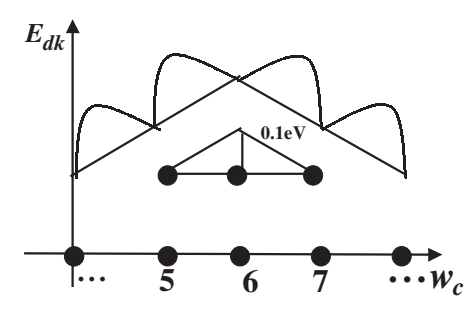

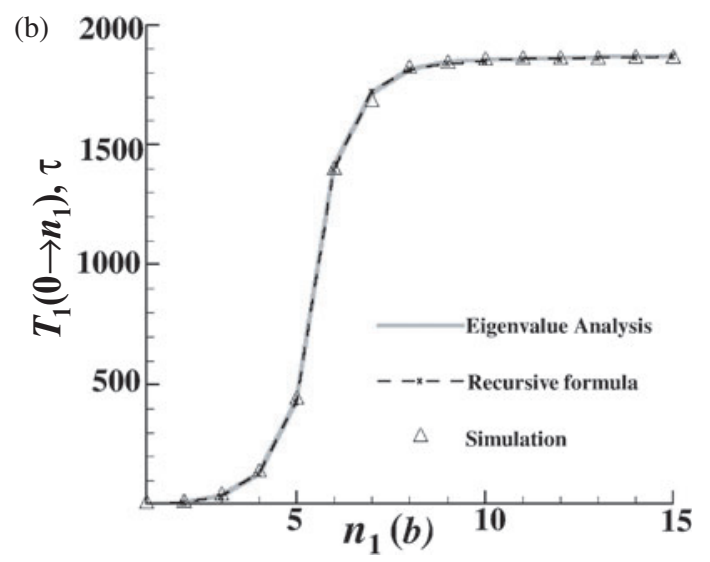

Figure 7. Idealized case 4: (a) the double kink energy separation relation for the case in which the energy of the double kink increases linearly with the double kink width to a critical size, beyond which it decreases linearly. $(b)$ The average first passage times $T_{1}\left(0 \rightarrow n_{1}\right)$ for the double kink to reach width $n_{1}$, where the lines and symbols have the same meaning as in figure $4(c)$.

an excellent approximation to the average first passage time for the case of linearly decreasing double kink energy.

\subsection{Case 4: triangular double kink energy separation profile}

We can combine cases 2 and 3 to consider the case in which the double kink energy increases with kink separation up to a critical width, and then decreases with increasing kink separation distance. The double kink energy-kink separation relationship is shown in figure 7( $a)$. This case corresponds to classical nucleation, where double kinks separated by less than a critical width tend to decay away, while double kinks separated by more than the critical separation grow. In this case, $w_{+}(i)$ is smaller than $w_{-}(i)$ for small kink separations and larger than $w_{-}(i)$ at large separations.

Figure $7(b)$ compares the average first passage time results from the two analytical approaches with the Monte Carlo simulation. We see that $T_{1}\left(0 \rightarrow n_{1}\right)$ increases nearly exponentially with increasing $n_{1}$ at small $n_{1}$ (well below the critical value of $n_{1}=6$ ) and then increases nearly linearly with increasing $n_{1}$ at large $n_{1}$ (well above the critical value of $n_{1}=6$ ). Results from the simulation and both analytical approaches are in very good agreement. Below the critical separation, the kinks attract one another. For these $n_{1}$ 's, the energy separation profile is identical to that in case 2, where the average first passage time increases exponentially with $n_{1}$. At kink separations greater than the critical width, the kinks repel. For these $n_{1}$ 's, the energy separation profile is identical to that in case 3 , where the average first passage time increases linearly with $n_{1}$. The results shown in figure $7(b)$ are consistent with these earlier observations except for double kink separations that are close to the critical separation $n_{1}=6$ (i.e. $5 \leqslant n_{1} \leqslant 8$ ).

\section{Screw dislocation in molybdenum}

The above case studies demonstrate that both first passage time analyses produce results that are in excellent agreement with the Monte Carlo simulation results. With the confidence gained from these case studies, we now examine the more realistic case of a kink motion 


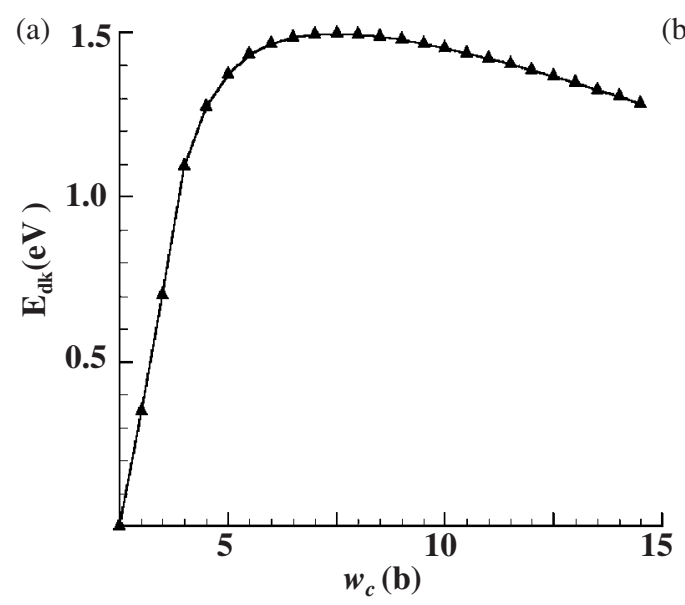

(b)
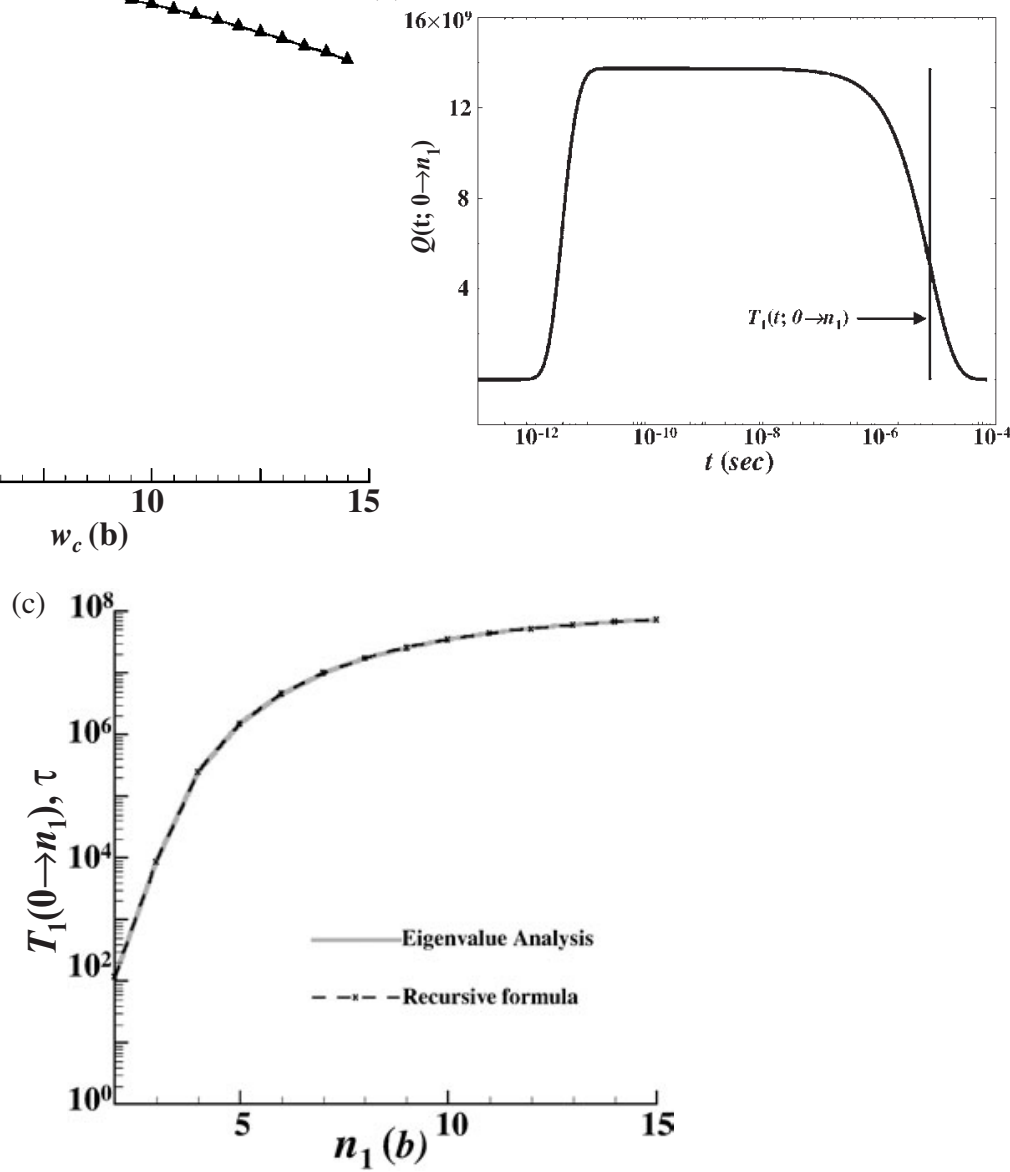

Figure 8. Double kink nucleation on a screw dislocation in molybdenum: $(a)$ the double kink energy as a function of kink separation. $(b)$ The distribution of first passage times for the double kink to reach a width of $15 b$. The vertical line indicates the average $T_{1}\left(0 \rightarrow n_{1}\right)$ of the distribution. (c) Average first passage times for double kink nucleation using the energy profile represented by $(a)$. The solid grey line shows the results of the eigenvalue approach and the dotted black line shows the results of the recursion formula analysis.

on a dislocation where the double kink separation profile has contributions from kink selfenergies, kink-kink elastic interactions and work term associated with an applied stress. For reasonable applied stresses, the time required for a Monte Carlo simulation to form and grow a double kink to a super-critical size is much too large to be practical. Hence, we only employ the two analytical methods described above and do not compare directly with Monte Carlo simulations. As a concrete example of a real dislocation, we consider double kink nucleation during the glide of a $\langle 111\rangle$ oriented screw dislocation in molybdenum with Burgers vector $\boldsymbol{b}=\left(a_{0} / 2\right)$ [111], where $a_{0}$ is the cubic lattice parameter. The free energy varies with double kink separation as [1]

$$
\Delta F=2 F_{\mathrm{k}}-\frac{\mu b^{2} h^{2}}{8 \pi w}-\sigma b h w,
$$


where $F_{\mathrm{k}}$ is the energy of an isolated kink, $w$ is the double kink width or kink separation, $\sigma$ is the applied stress (see above), $h$ is the kink height and $\mu$ is the shear modulus.

We consider double kink nucleation on a screw dislocation in Mo with an external stress of $100 \mathrm{MPa}$. The variation of the energy of a double kink on a screw dislocation in molybdenum with kink separation for an external applied stress of $100 \mathrm{MPa}$ is plotted in figure $8(a)$ using data obtained from the literature $[1,7,9]$. The probability that a double kink expands to a critical kink separation is very low because of kink-kink attraction. Thus double kinks with small kink separations tend to annihilate immediately after nucleation. For this applied stress, the critical kink separation $w^{*}$ is approximately $7 b$. In the present analyses, we determine the first passage time for a kink separation of $15 b$.

Figure $8(b)$ shows the distribution of the first passage times $Q(t ; 0 \rightarrow 15)$ for a double kink of $15 \mathrm{~b}$ determined from equations (12) at $T=1000 \mathrm{~K}$. The first passage times are distributed over a very large timescale (approximately five orders of magnitude). The average first passage time is $7 \mu \mathrm{s}$. Rather than simply using the average first passage time, the first passage time should be randomly chosen from the distribution $Q(t ; 0 \rightarrow 15)$. A kMC simulation of this process would require a time step of order $7 \mathrm{ps}$. Therefore, inserting a double kink with a separation of $15 b$ and adjusting the kMC simulation clock, rather than forming one with a separation of $b$ and waiting for it to grow to $15 b$, requires a factor of approximately $10^{6}$ fewer MC steps. Even with the overhead associated with calculating the distribution of first passage times, this should accelerate the kMC simulation by a factor of at least $10^{4}$. The kMC simulation could be accelerated even further by replacing the true distribution of first passage times with the mean first passage time, determined using the recursion formula, equations (13) and (14). Figure 8(c) demonstrates that the average first passage time for the conditions in figures $8(a)$ and $(b)$ determined using the full eigenvalue equation and the recursion formula yield very similar results (the per cent error in replacing the full eigenvalue result with the simpler method is approximately $0.1 \%$ ).

The rate of double kink nucleation depends on both the applied external stress and the temperature. Figure 9(a) shows the variation of stable double kink nucleation rate with applied stress and figure $9(b)$ shows the variation of stable double kink nucleation rate with temperature. The double kink nucleation rate increases with increasing external stress and with increasing temperature. Increasing the external stress effectively lowers the barrier in figure $8(a)$, while increasing the temperature decreases the time required to obtain a thermal fluctuation needed to overcome the barrier.

\section{Discussion and conclusion}

The first passage time analysis presented above can be applied in the context of kMC simulations where there is a clear separation of timescales between different types of events. Such situations frequently arise in kMC simulations of a wide variety of materials phenomena in addition to the double kink nucleation example discussed above.

As another example of this, consider the kMC simulation of the chemical vapour deposition of $\{111\}$ oriented diamond [11] under conditions that are of hot filament and microwave plasma CVD reactor environments. The diamond surface evolves by the chemisorption, desorption/abstraction and incorporation of molecular species. The gas phase in the CVD reactor commonly contains $\mathrm{H}, \mathrm{H}_{2}$ and various hydrocarbons. Under typical growth conditions, the diamond surface is largely covered with a passivating layer of $\mathrm{H}$ atoms. Surface sites are activated by $\mathrm{H}$ desorption or abstraction which leaves an open surface site where hydrocarbons can attach. Typically, such open surface sites are repassivated by $\mathrm{H}$ adsorption before any hydrocarbon reaches that site. The diamond surface is extremely dynamic with rapid $\mathrm{H}$ 

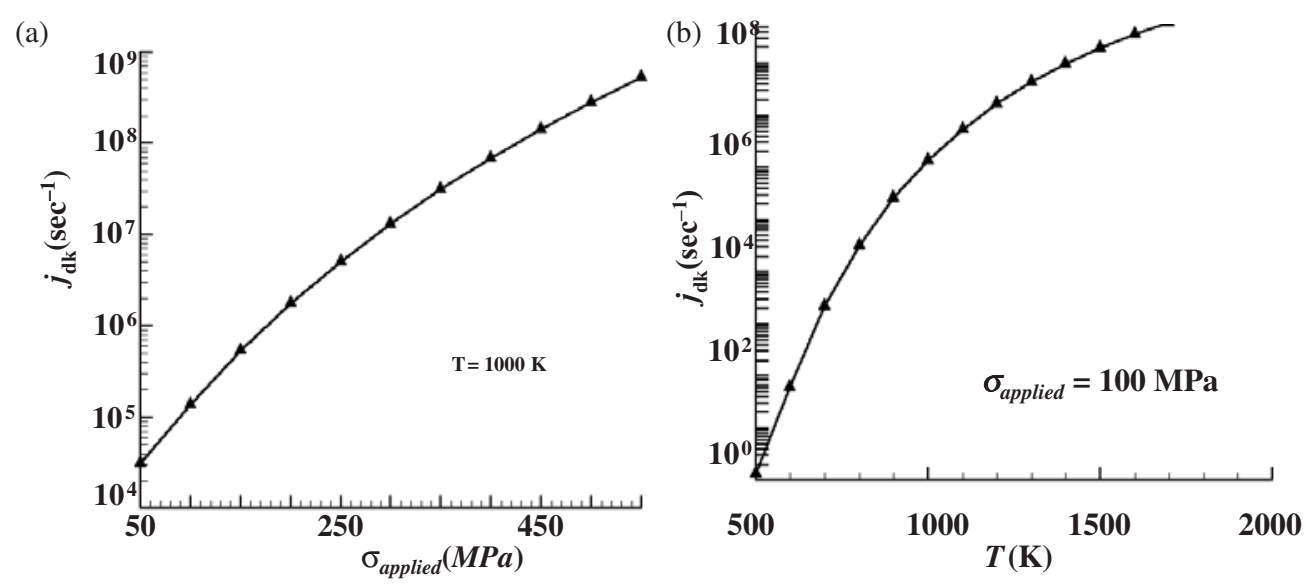

Figure 9. The average nucleation rates $j_{\mathrm{dk}}$ of double kinks on a screw dislocation in molybdenum as a function of $(a)$ applied stress $\sigma_{\text {applied }}(\mathrm{MPa})$ and $(b)$ temperature $T(\mathrm{~K})$.

adsorption, desorption and abstraction which occurs on timescales much faster than any other surface event. The growth of a new $\{111\}$ layer diamond on a flat $\{111\}$ surface requires the formation of a 3-carbon nucleus (island). However, deposition of three adjacent carbons on the surface before any of them desorb is very rare. The simulation, therefore, uses a large number of Monte Carlo steps simulating sub-critical nucleation events that involve adsorption and desorption of $\mathrm{H}$ as well as carbon containing radicals such as $\mathrm{CH}_{3}, \mathrm{C}_{2} \mathrm{H}_{2}$ which desorb prior to nucleation. Under typical growth conditions $\left(T=1200 \mathrm{~K}\right.$, partial pressures $P_{\mathrm{H}}=0.04$ Torr, $P_{\mathrm{H}_{2}}=18$ Torr, $P_{\mathrm{CH}_{3}}=0.004$ Torr and $P_{\mathrm{C}_{2} \mathrm{H}_{2}}=0.04$ Torr), it takes $10^{5}$ Monte Carlo steps to simulate the nucleation of a stable island of carbon atoms on the diamond film surface. In such a case, the first passage time analysis can be used to improve the computational efficiency of the kMC simulation by calculating the consolidated rate for a stable nucleus of carbon atoms. A first passage time analysis could include all of the H-events leading up to the insertion of a hydrocarbon or all of the events leading up to the formation of a critical carbon island nucleus on the surface. This elimination of the hydrogen abstraction-adsorption reactions from the simulation (which do not contribute to the overall growth of the diamond film) would lead to $\mathrm{kMC}$ simulations that are as much as $10^{4}$ times faster than standard methods [11]. This type of gain in efficiency would not be atypical for $\mathrm{kMC}$ simulations involving nucleation events or other rare events.

In this paper, we have described how the first passage time analysis may be used to obtain consolidated rates of rare events; i.e. the nucleation of double kinks during dislocation glide in the present case. The double kink nucleation process is treated as a temporally homogeneous birth-death Markov process. We analyse the Markov process to obtain the distribution and the average of first passage times for the nucleation of a double kink of a particular size (critical kink separation). We have discussed two methods to determine the first passage time; one computes the distribution and the average of the first passage times (the eigenvalue approach), while the other uses a recursive relation to calculate the average first passage time (the recursion formula). The average first passage times calculated by both approaches were compared with the results of $\mathrm{kMC}$ simulations for four idealized cases of double kink nucleation. We found that the first passage time analyses were both in excellent agreement with Monte Carlo simulation results. Finally, we applied this method to the realistic case of double kink nucleation on a screw dislocation in molybdenum. This results in very efficient predictions of double kink 
nucleation rates as a function of applied stress and temperature. The present approach of nucleating a critical double kink on an otherwise straight dislocation allows us to increase the computational efficiency of the kMC simulations of dislocation migration by a factor of $10^{6}$ compared with the more straightforward kMC simulations that account for double kink formation from a straight dislocation line and allowing it to grow to the critical size.

\section{Acknowledgments}

The authors gratefully acknowledge useful discussions with W Cai, V Bulatov, M Kalos, D Mason and M Mendelev. Part of this work was performed at the Materials Research Institute (MRI) at the Lawrence Livermore National Laboratory as part of the Computational Materials Science Summer School (2001). This research was supported by the Lawrence Livermore National Laboratory.

\section{References}

[1] Hirth J P and Lothe J 1982 Theory of Dislocations 2nd edn (New York: Wiley)

[2] Bortz A B, Kalos M H and Lebowitz J L 1975 J. Comput. Phys. 17 10-18

[3] Fichthorn K A and Weinberg W H 1991 J. Chem. Phys. 95 1090-6

[4] Cai W, Bulatov V and Yip S 1999 J. Comput. Aided Mater. Design 6 175-83

[5] Lin K and Chrzan D C 1999 Phys. Rev. B 60 3799-805

[6] Tang M, Kubin L P and Canova G R 1998 Acta Materialia 46 3221-5

[7] Cai W, Bulatov V, Yip S and Argon A S 2001 Mater. Sci. and Eng. A 309 270-3

[8] Gillespie D T 1992 Markov Processes: An Introduction for Physical Scientists (San Diego, CA: Academic)

[9] Xu W and Moriarty J A 1998 Comput. Mater. Sci. $9348-56$

[10] Gillespie D T 1982 J. Chem. Phys. 76 3762-7

[11] Battaile C C, Srolovitz D J and Butler J E 1998 J. Appl. Phys. 826293

[12] Montroll E W and Shlesinger M F 1984 The wonderful world of random walks Nonequilibium Phenomena II: From Stochastics to Hydrodynamics ed J L Lebowitz and E W Montroll (Amsterdam: North-Holland) pp 1-121

[13] Karlin S and Taylor H M 1975 A First Course in Stochastic Simulations 2nd edn (New York: Academic) 